\title{
Design of the third-generation neutron spallation target for the CERN's n_TOF facility
}

\author{
Raffaele Esposito ${ }^{\mathrm{a}, \mathrm{b}, *}$ and Marco Calviani ${ }^{\mathrm{a}, * *}$ \\ ${ }^{a}$ European Laboratory for Particle Physics (CERN), Switzerland \\ E-mails: raffaele.esposito@cern.ch, marco.calviani@cern.ch \\ ${ }^{\mathrm{b}}$ École Polytechnique fédérale de Lausanne (EPFL), Switzerland
}

\begin{abstract}
The neutron Time-Of-Flight (n_TOF) facility at the European Laboratory for Particle Physics (CERN) is a pulsed white-spectrum neutron spallation source producing neutrons for two experimental areas: EAR1, located $185 \mathrm{~m}$ downstream of the spallation target, and EAR2, located $20 \mathrm{~m}$ above the target. The facility is based on a lead target impacted by a high-intensity $20 \mathrm{GeV} / \mathrm{c}$ proton beam. It is designed to study neutron-nucleus interactions for neutron kinetic energies from a few $\mathrm{meV}$ to several $\mathrm{GeV}$, with applications in nuclear astrophysics, nuclear technology, and medical research. The facility is undergoing a major upgrade in 2019-2020, which will include the installation of the new third-generation target. The second-generation target consists in a water-cooled lead cylinder, while the new target will be cooled by nitrogen to avoid erosion-corrosion phenomena and contamination of the cooling water with radioactive lead spallation products. The new design will be optimized also for the vertical flight path. The operation of the new spallation target will start in 2021. This paper presents an overview on the evolution of the design and on the related R\&D activities (including beam irradiation tests) carried out to ensure the best performance for both experimental areas and avoid the contamination issues of the previous targets.
\end{abstract}

Keywords: Neutron, spallation, target, source, n_TOF

\section{Introduction: The n_TOF facility at CERN}

The neutron Time-Of-Flight (n_TOF) facility at the European Laboratory for Particle Physics (CERN) is composed of a spallation neutron source coupled to two neutron flight paths. Neutron energies are deduced from the time of flight between source and detector, hence the name of the facility. The neutron source is driven by a pulsed proton beam extracted from the Proton Synchrotron (PS) machine of the CERN's accelerator complex. Up to $8.5 \times 10^{12}$ protons with a momentum of $20 \mathrm{GeV} / c$ are extracted from the PS with a minimum repetition rate of $1.2 \mathrm{~s}$ and impact a pure lead target in a single pulse of $6 \mathrm{~ns}$ (RMS). Every proton yields about 300 neutrons. The initially fast neutron spectrum is moderated by water before being collimated and transported through beam pipes to two experimental areas: EAR1, located $185 \mathrm{~m}$ downstream of the spallation target, and EAR2, at $20 \mathrm{~m}$ above the target (Fig. 1). Both experimental areas are classified as Class-A laboratories, i.e. capable of dealing with unsealed samples $[19,20]$.

The $\mathrm{n}_{-} \mathrm{TOF}$ facility is an important tool for studies of neutron-induced reactions, especially for radioactive samples. The $\mathrm{n}$-TOF collaboration is composed of 42 member institutions from Europe, India, Japan, Russia, and Australia. The research purposes include the fields of nuclear physics and astrophysics (Big Bang and stellar nucleosynthesis [5,15]), nuclear technologies (nuclear energy production and waste management $[1,18]$ ), nuclear

\footnotetext{
*Corresponding author. E-mail: raffaele.esposito@cern.ch.

***For the n_TOF Target \#3 Project Team.
} 


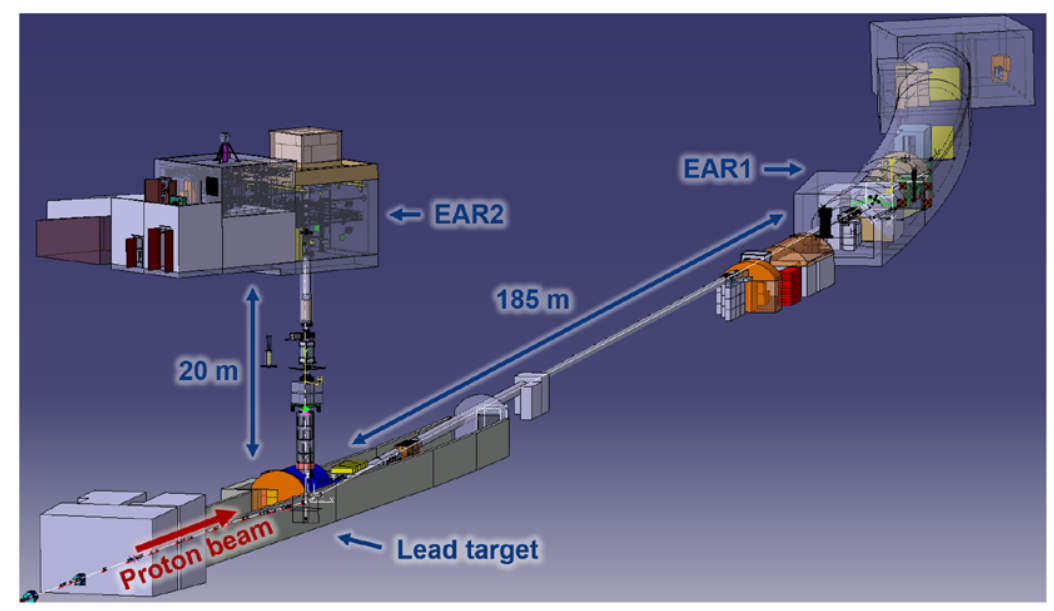

Fig. 1. n_TOF facility layout. A $20 \mathrm{GeV} / \mathrm{c}$ proton beam from the Proton Synchrotron (PS) impacts a pure lead target. The generated neutrons reach two experimental areas: EAR1, $185 \mathrm{~m}$ downstream of the target, and EAR2, at $20 \mathrm{~m}$ above the target.

medicine (neutron capture therapy) as well as neutron imaging (including the inspection of highly-activated samples [21]).

The relevant features of the neutron source are:

- Wide energy range: from thermal to GeV.

- Low repetition rate: 1 pulse every $1.2 \mathrm{~s}$ (no superposition between neutrons from different proton pulses).

- High energy resolution: $\Delta E / E=10^{-4}$ for EAR1 and $\Delta E / E=10^{-3}$ for EAR2 (at $10 \mathrm{keV}$ ).

- High instantaneous neutron flux: $2 \times 10^{5} \mathrm{n} / \mathrm{cm}^{2} /$ pulse for EAR1 and $3 \times 10^{6} \mathrm{n} / \mathrm{cm}^{2} /$ pulse for EAR2. The acquisition stays open only for $80 \mathrm{~ms}$ in EAR1 and $10 \mathrm{~ms}$ in EAR2 (time of flight of thermal neutrons).

Thanks to these features (especially the very high instantaneous intensity), the facility is well suited to perform high-precision measurements of neutron cross-sections and to study radioactive isotopes with very low crosssections, or available in only small quantities, or with very high specific activity.

During CERN's Long Shutdown 2 (LS2), the facility is undergoing an important consolidation and upgrade, which - amongst other activities - includes the construction and installation of the new third-generation spallation target.

\section{History of the facility}

\subsection{Target \#1 (2000-2004)}

The n_TOF facility was conceived to perform accurate neutron cross-section measurements for different purposes [22]: design of Accelerator Driven Systems (ADS), transmutation of radioactive waste, astrophysics research (synthesis of heavy elements in the Universe), and advancement in fundamental knowledge of nuclear physics.

The facility was commissioned in 2000, with the $185 \mathrm{~m}$ horizontal flight path. The first-generation target (Target \#1, Fig. 2) was made of a series of pure lead bricks [2], stacked together to form a massive block $\left(80 \times 80 \times 40 \mathrm{~cm}^{3}\right)$.

The target was designed to receive 4 pulses of $7 \times 10^{12}$ protons every $14.4 \mathrm{~s}$, spaced by at least $1.2 \mathrm{~s}$. The pulse duration was $6 \mathrm{~ns}$ (RMS) and the beam size $7.8 \times 5.6 \mathrm{~mm}^{2}(1 \sigma)$. The target was cooled by a demineralised water flow of $22 \mathrm{~m}^{3} / \mathrm{h}$ at $30^{\circ} \mathrm{C}$. The water layer downstream of the target was $5 \mathrm{~cm}$ thick and had also the function of neutron moderator. Once the periodic regime is reached, a peak temperature equal to $165^{\circ} \mathrm{C}$ has been estimated by means of finite-element thermal simulations. Six thermocouples were fixed on the target to monitor its temperature and confirm the expected thermal response [7]. 

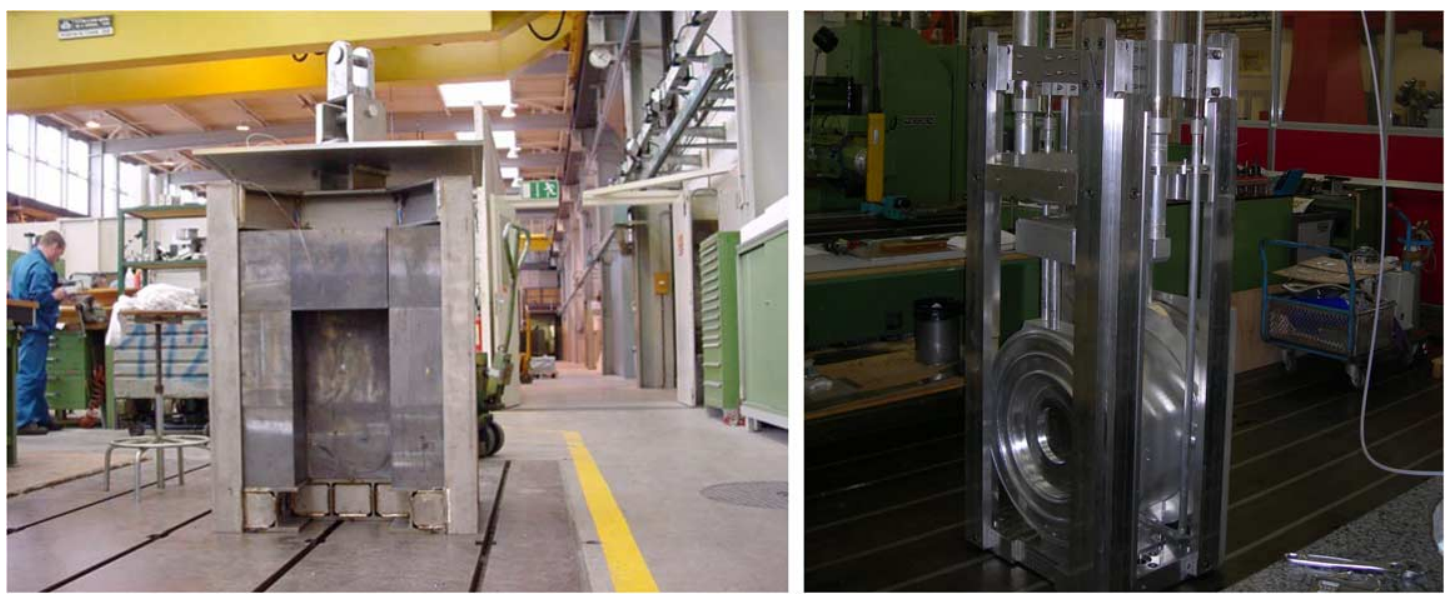

Fig. 2. Left: Target \#1, composed of a water-cooled pile of lead bricks [2]. Right: Target \#2, consisting in a water-cooled lead cylinder [9].

In 2004, after three years of successful operation, an abnormal increase of radioactivity in the cooling circuit was observed. The issue was ascribed to inefficient cooling at the beam impinging point and lack of chemical control of the cooling water, facilitating pitting corrosion and contamination of water with radioactive lead spallation products [3].

\subsection{Target \#2 (2008-2019)}

A second-generation target was then designed (Target \#2), aimed at addressing the operational concerns of the first-generation target [9]. Target \#2 is a cylinder with diameter of $60 \mathrm{~cm}$ and axial length of $40 \mathrm{~cm}$, cooled by a forced flow of water $\left(7 \mathrm{~m}^{3} / \mathrm{h}\right)$ at $0.5 \mathrm{barg}$. A separate moderator assembly, made of aluminum alloy, has been added to have a fluid for moderation different than the one of the cooling circuit. The moderating fluid was switched to borated water (1.28 wt\%), which reduced the background for the neutron capture measurements in EAR1 [4]. The new target was enclosed in an aluminum vessel and it was slightly smaller than Target \#1, so the entire assembly fitted into the old target container, which acted as secondary containment vessel.

The target was installed at the end of 2008 and started operation in early 2009. Some critical aspects of the design were highlighted, like the very low thickness $(600 \mu \mathrm{m})$ of the aluminium window separating the cooling and the moderator circuits. This window needed to be thin to reduce photon background as much as possible in the experimental areas, but it was considered a risk for corrosion and failure in the long term [10].

Another critical aspect was that direct contact between pure lead and cooling water was still present, with the risk of erosion-corrosion phenomena and contamination of the cooling water with radioactive spallation products from lead. Different measures were taken to reduce this risk. The circulation of cooling water was improved, the average beam intensity limited to $1.67 \times 10^{12} \mathrm{p}^{+} / \mathrm{s}$ and the beam was defocused. Moreover, the water chemistry was controlled and kept within very tight operational constraints to minimize its corrosion capacity [10].

Due to these critical aspects, the target was produced and installed considering an estimated lifetime of about ten years. In the last years of target operation, an increase in the cooling water activity was detected and first signs of corrosion of the neutron window were also observed by endoscopic inspection, confirming the necessity of replacing the target with a new one [13].

In 2014 the new experimental area (EAR2) was built $20 \mathrm{~m}$ above the target [14]. Even if the design included the possibility of inserting a vacuum tube on top of the spallation target, the resolution in EAR2 was not optimal, since the target shape was not conceived for this experimental area: the moderation of the neutrons directed upwards was achieved with the cooling-water volume above the lead target core, which had an irregular shape. 


\section{Target \#3: Evolution of the design}

The design of the new-generation spallation target (Target \#3) began in 2016 with preliminary studies of different target concepts [10].

The objectives to be pursued by the new design were:

- Limiting erosion-corrosion phenomena and contamination of the cooling water with lead spallation products.

- Improving neutron fluence and resolution for EAR2, thanks to the addition of a dedicated moderator.

- Allowing a higher beam pulse intensity to be accepted (from $7 \times 10^{12}$ to $10^{13}$ protons-per-pulse).

- Preserving the excellent physics performances at EAR1.

- Preserving or improving background conditions in the experimental areas.

In the preliminary design stage, six different concepts were evaluated. Based on these studies, two more concepts (for a total of eight) were derived from the preliminary ones, including the final design choice. Figure 3 compares the neutron fluence to EAR1 downstream of the target with respect to Target \#2, estimated by FLUKA Monte Carlo code $[6,17]$ for the six preliminary solutions. Figure 4 shows a similar comparison between the three most promising solutions with respect to Target \#2. The data in Fig. 3 is extracted right after the target for quicker preliminary simulations, while the data in Fig. 4 is extracted at the EAR1 to show a comparison that is more meaningful for the final decision.

(1) Target \#2: pure lead cylinder, $60 \mathrm{~cm}$ in diameter and $40 \mathrm{~cm}$ in length. This concept is reported as reference.

(2) Pb cylinder with flat top. Similar to Target \#2, this solution had the only objective of improving the resolution to EAR2 without changing anything else. The distance between the cylinder axis and the horizontal cut was fixed to $15 \mathrm{~cm}$ after optimization studies directed to find the best compromise in terms of neutron fluence between EAR1 and EAR2. The erosion-corrosion and contamination issues were not addressed by this solution, which was considered a backup choice.

(3) Ta-cladded W surrounded by a W mass. A cylinder of tungsten $(\varnothing 10 \times 25 \mathrm{~cm})$ with a Ta cladding obtained by hot isostatic pressing (HIP), enclosed in a massive tungsten block. The Ta-W core would be the part directly impacted by beam, and the only one to be actively water cooled. The excellent corrosion resistance of tantalum would solve the contamination issues, while the better thermo-mechanical properties of tungsten and tantalum (compared to lead) would eliminate any thermo-structural concern even for higher average power on target. Moreover, the HIP process would provide an excellent thermal conductance at the Ta-W interface and a lot of experience had already been acquired at CERN on R\&D of HIPed Ta-W assemblies [8].

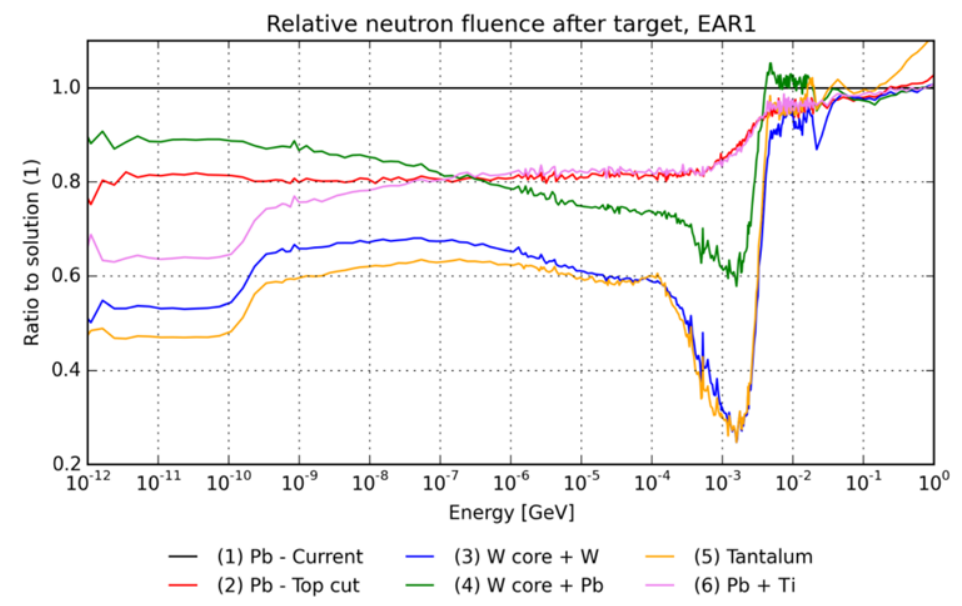

Fig. 3. The plot shows the neutron fluence towards the first experimental area (EAR1) right after the target, for the six preliminary design proposals. The neutron fluence has been estimated by means of FLUKA Monte Carlo code [10]. 


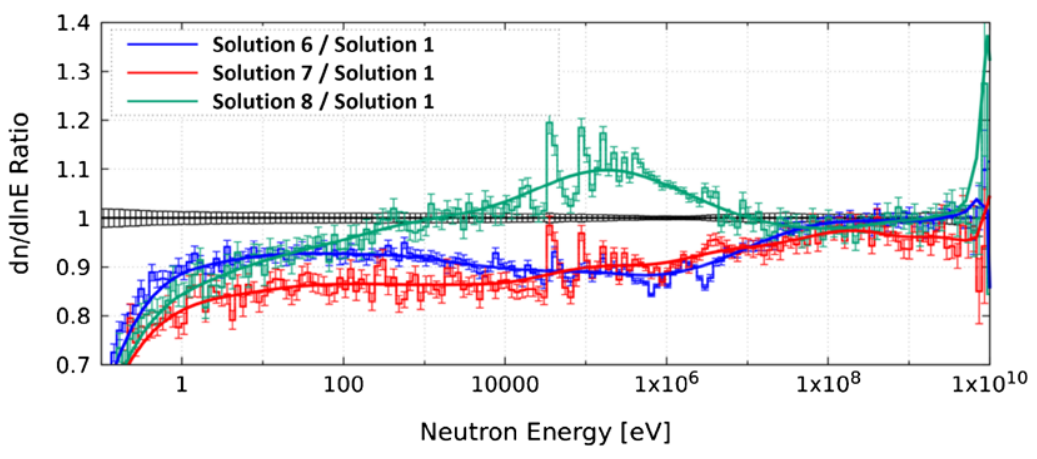

Fig. 4. Neutron fluence at EAR1 of the three most promising solutions with respect to Target \#2.
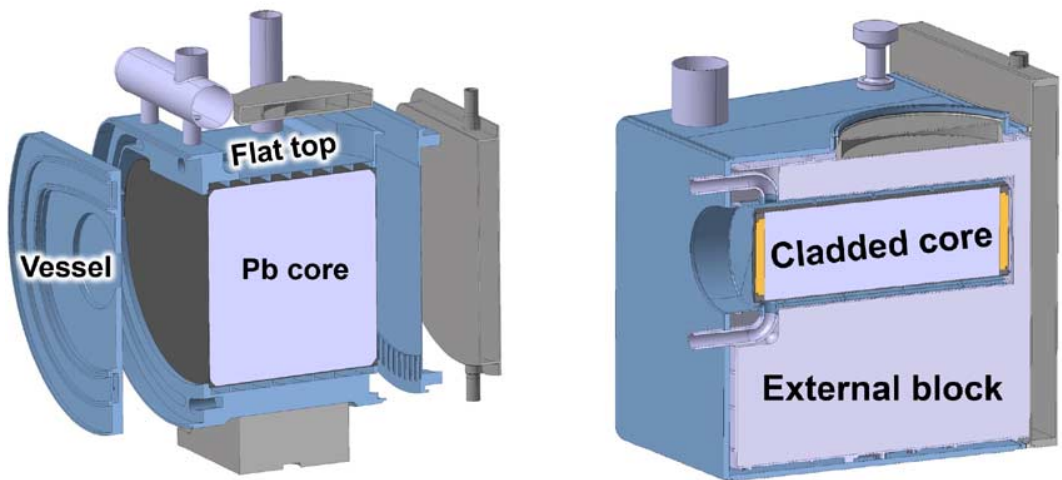

Fig. 5. Left: water-cooled lead cylinder with flat top, concept of solutions (2) and (6). Right: water-cooled cladded core with gas-cooled external mass, concept of solutions (3), (4), and upgrades [10,11].

For the external tungsten mass, a gas circuit or even natural convection would suffice for an adequate cooling. As is shown in Fig. 3, the neutron fluence of this solution is only 25-30\% the one of Target \#2 around $1 \mathrm{MeV}$ (evaporation peak), and about 60-70\% from $1 \mathrm{keV}$ to $100 \mathrm{keV}$. Therefore, this solution was discarded.

(4) Ta-cladded W surrounded by a Pb mass. This design solution was similar to solution (3), with the only difference being the external mass made of lead instead of tungsten. This variation should provide better physics performances in terms of neutron production and background, due to the higher inelastic crosssection of $\mathrm{W}$ with respect to pure $\mathrm{Pb}$. The neutron fluence (Fig. 3) is equal to 60-65\% the one of Target \#2 around $1 \mathrm{MeV}$. The reduction is due to the strong inelastic channels in tungsten around a few hundred $\mathrm{keV}-1 \mathrm{MeV}$. This design was improved with additional lead downstream of the Ta-W core, which reduced background and increased neutron fluence. Despite the effort, neutron fluence was still 25\% lower than Target $\# 2$ at $1 \mathrm{MeV}$, and the solution was discarded due to worse physics performances when compared to other solutions.

(5) Ta target. This concept consisted in a $50 \times 50 \times 22.5 \mathrm{~cm}^{3}$ water-cooled volume of tantalum, divided into three slices to increase the surface exposed to the coolant. This solution was technically very simple, although very expensive due to the high cost of the base material. Moreover, the neutron fluence of this solution (Fig. 3) is only $20-30 \%$ the one of Target \#2 around $1 \mathrm{MeV}$, and about $60-65 \%$ from $1 \mathrm{keV}$ to $100 \mathrm{keV}$. Therefore, this solution was also discarded.

(6) Ti-6Al-4V-cladded $P b$. This design was similar to solution (2), with the addition of a titanium Grade 5 layer protecting the lead core from water corrosion (Fig. 5). The choice of the cladding material was the result of an optimization procedure directed to find the best mix of neutron flux, background, elasticity, corrosion resistance, yield and fatigue strength compared to the arising stress. The materials considered in the selection 
of the cladding layer included: tantalum, niobium, Zircaloy, stainless steel, Inconel, and Nitronic. The main issue of this solution was the uncertainty related to the thermal contact at the interface between lead and Ti-6Al-4V: extensive R\&D and prototyping activities have been carried out to find the best manufacturing process that could ensure an acceptable thermal conductance at the interface. This solution was characterized by almost the same EAR1 neutron fluence and higher EAR2 neutron fluence than Target \#2 (Fig. 4), but also by a significantly higher background caused by the presence of titanium downstream of the lead core. To avoid an excessive increase of background and to be compatible with the EAR1 performances of the secondgeneration target, the Ti-6Al-4V layer should have been $0.5-1 \mathrm{~mm}$ thick. Considering that the Ti-6Al-4V layer is $600 \mathrm{~mm}$ wide, manufacturing and assembly of this solution turned out to be particularly difficult. Moreover, during operation, the lead core would swell due to thermal expansion and push the Ti-6Al-4V layer from inside. Such a wide and thin layer of Ti-6Al-4V would have elastic deformations in the order of centimetres, incompatible with the assembly. Large deformations of the core would also change the cooling water volume surrounding the target, worsening the resolution. In addition, this behavior would provoke loss of contact between the lead core and the Ti-6Al-4V layer, making the target cooling largely inefficient.

(7) Ti-6Al-4V-cladded Pb surrounded by a Pb mass. A new design was then proposed, merging solution (4) and (6) and keeping the concept of a water-cooled core subject to the direct impact of the beam and surrounded by an external massive lead block [11]. The Ta-W core of solution (4) is replaced, in this new concept, by a Ti-6Al-4V-cladded-Pb core as in solution (6). In this case, the Ti-6Al-4V-cladded-Pb part can be perfectly cylindrical and much smaller $(\varnothing 15 \times 30 \mathrm{~cm})$, thus removing the manufacturing and assembling issues. Cooling water flows inside a thin stainless steel vessel hosting the Ti-6Al-4V-cladded-Pb core. The absence of tungsten in this solution removes the issue related to the reduction of neutron flux (Fig. 4), while the presence of lead downstream of the Ti-6Al-4V-cladded-Pb core helps decreasing the background to levels even lower than in Target \#2. The lids of the Ti-6Al-4V cladding should still be very thin $(500 \mu \mathrm{m})$ to keep the background low. Therefore, they undergo large deformations, even if smaller than in solution (6) thanks to their smaller size $(15 \mathrm{~cm}$ instead of $60 \mathrm{~cm})$. This issue was solved adding, inside the Ti-6Al-4V envelope, two 15$\mathrm{mm}$ thick beryllium stiffening plates. These plates reduce the deflection of the Ti-6Al-4V lids and increase the contact pressure at the interfaces, thus improving the thermal conductance [11]. A real-scale prototype of the Ti-6Al-4V-cladded-Pb core has been manufactured and tested under proton-beam pulses in the CERN's HiRadMat facility [16], validating the structural robustness of the design concept. The main drawback of this design is the complexity: the final assembly is made of many parts and different materials, increasing risks and potential failures. Moreover, the external lead block must be actively gas cooled, thus two cooling systems working in parallel would be needed (water and gas), increasing the operational complexity of the target assembly.

(8) $\mathrm{N}_{2}$-cooled $\mathrm{Pb}$ slices. A simpler solution, in terms of manufacturing and assembling, was considered in the last stage of this iterative design process. This alternative concept is an upgrade of the previously mentioned solution (2), and consists in six pure-lead slices cooled by a forced flow of gaseous nitrogen. After three design reviews, this new concept was favoured to become the baseline design for being more essential and providing better physics performances in terms of neutron flux (Fig. 4), background and resolution [12]. This solution has also been tested and validated under proton-beam irradiation in the HiRadMat facility. The detailed design is presented in Section 4.

\section{Target \#3 design}

The final design of the n_TOF Target \#3 is based on six pure lead slices, as shown in Fig. 6. To ensure that lead impurities will have a negligible effect on physics performances, the base lead material has a minimum purity of $99.9 \mathrm{wt} \%$. Shape and size of the slices have been defined after an optimization procedure directed to find the best compromise between the neutron flux towards EAR1 and EAR2, as well as to keep the background low. The slices are $60 \mathrm{~cm}$ wide and tall, and $5 \mathrm{~cm}$ thick. The only exception is the last slice along the beam direction, which is $15 \mathrm{~cm}$ thick to keep the background low. The flat top helps improving the resolution for EAR2 [12]. 


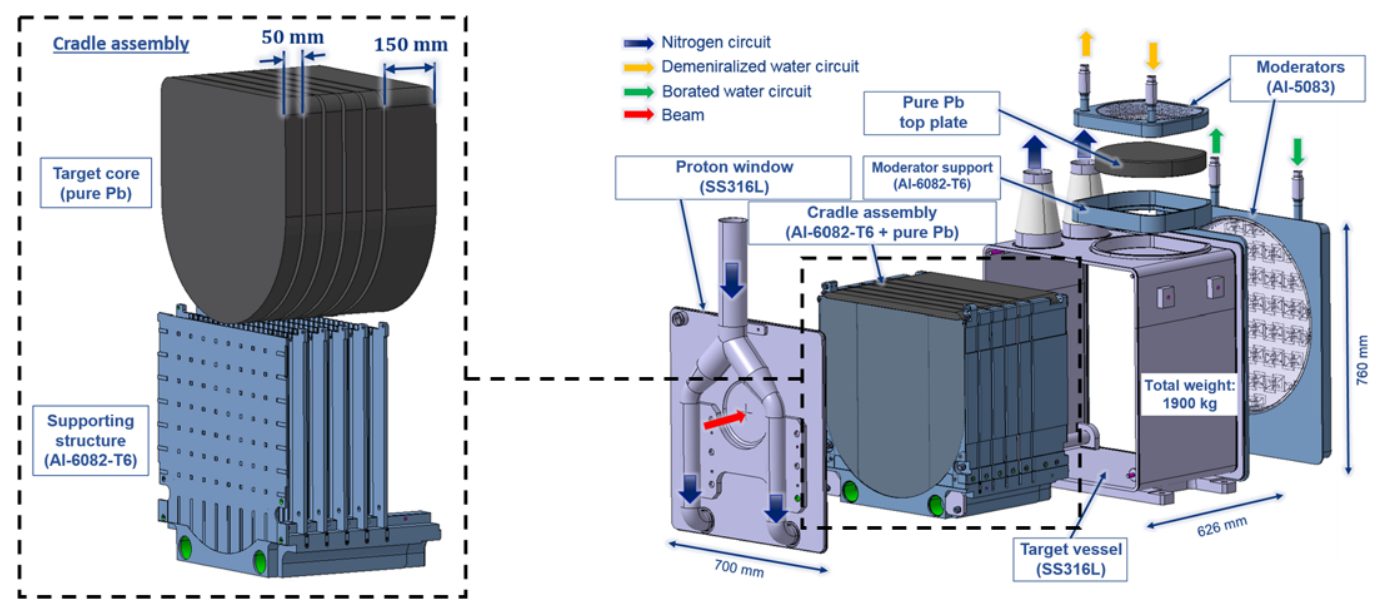

Fig. 6. Exploded view (3D model) of the n_TOF Target \#3 final design [12]. Left: cradle assembly. Right: whole target assembly.

The lead slices are cooled down by a forced flow of pure nitrogen. Switching from the water cooling of Target \#2 to nitrogen cooling in Target \#3 reduced the heat removed per unit area. To keep the same peak temperature in the new target, the surface exposed to the coolant must be increased, hence the slicing. The upgrade to nitrogen cooling avoids water contamination issues, while the absence of cladding materials keeps the background low. In case undesired interactions between nitrogen and lead arise during operation, the cooling system can also work with inert gas (argon) at the cost of a slightly lower cooling efficiency.

This solution is characterized by the best physics performances (neutron flux, background, and resolution) among the ones presented in Section 3 that solve the contamination issues (Fig. 4).

Figure 6 shows the target assembly. The lead slices are supported by an aluminium-alloy structure (Al-6082-T6). This structure has different functions:

- supports the lead slices and holds them in place in case of shocks or damage;

- regulates the nitrogen flow with cooling channels and constrictions opportunely machined on the aluminum plates;

- avoids plastic flow of lead due to creep effects.

The sub-assembly composed of lead slices and aluminum supporting structure is hosted inside a stainless-steel 316L vessel with low cobalt content (around $0.078 \%$ with the final batches acquired for production) to reduce the residual dose around the target. The upstream face of the vessel includes a thin window at the beam-impinging location to avoid interaction between proton beam and stainless-steel vessel.

Figure 6 also shows the two moderators, one for each experimental area: the EAR2 moderator is mounted outside of the stainless-steel vessel, above the target, while the EAR1 moderator is integrated into it and represents its downstream face. The two moderator casings are made of aluminum alloy 5083. The bond between the EAR1 moderator and the stainless-steel vessel is realized with a bimetallic transition obtained by a process of explosion cladding. Mechanical tests have been performed on explosion-cladded bonds from different suppliers to validate the solution. The choice of stainless-steel for the vessel instead of aluminum (as in the moderator casings) was requested by the radiation protection team to enhance compatibility with the radioactive wate matrix.

An additional lead plate, shown in Fig. 6 between the stainless-steel vessel and the EAR2 moderator, has been included to reduce the photon background towards EAR2.

The beam parameters representing the worst-case scenario and considered for the design are listed below:

- Momentum: $20 \mathrm{GeV} / c$.

- Protons per pulse: $10^{13}$. 

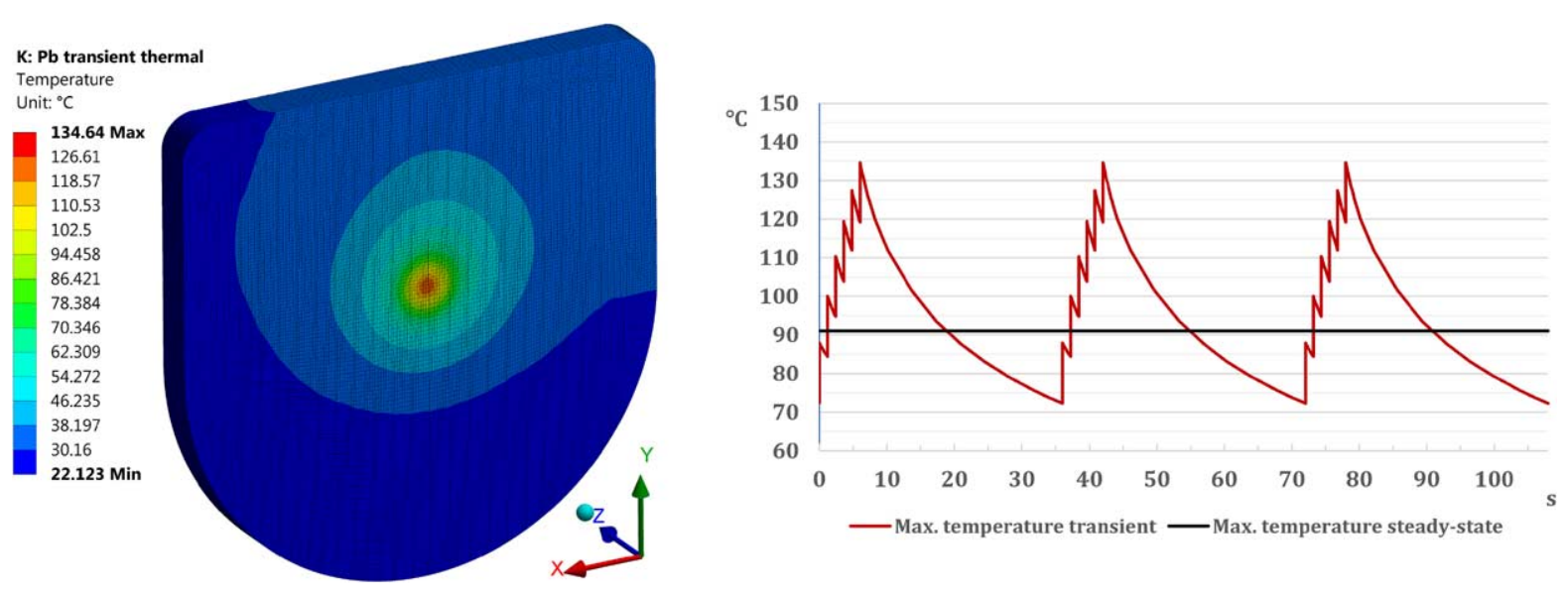

Fig. 7. Thermal FEM studies of Target \#3. Left: peak temperature distribution in the second $\mathrm{Pb}$ slice. Right: transients of maximum temperature at periodic regime; the steady-state average is also shown in the plot [12].

- Energy in one pulse: $32 \mathrm{~kJ}$.

- Pulse duration: 6 ns $(1 \sigma)$.

- Peak power: $1.8 \mathrm{TW}$.

- Time structure: 6 pulses every $1.2 \mathrm{~s}$ followed by $30 \mathrm{~s}$ of cool-down, for a supercycle duration totaling $36 \mathrm{~s}$ (Fig. 7).

About half of the specified energy and power is absorbed by the target. The target should be able to operate for ten years, until CERN's Long Shutdown 4 (currently foreseen around 2030). The total number of high intensity pulses impacting the target during its lifetime can be conservatively assumed, for structural fatigue evaluations, equal to $3 \times 10^{7}$.

The particle beam impacts the target with a tilt of $10^{\circ}$ in the horizontal plane to reduce the EAR1 background caused by $\gamma$-rays and high-energy charged particles [2].

Thermo-mechanical and Computational Fluid Dynamics (CFD) simulations predict the highest temperature to arise in the second lead slice (along the beam direction) and reach $135^{\circ} \mathrm{C}$ (Fig. 7), safely below the lead melting temperature $\left(327^{\circ} \mathrm{C}\right)$ but high enough to provoke reduction in yield strength and creep effects.

The most critical aspect of the target thermo-mechanical response is related to the impulsive character of the thermal load. The quick raise of temperature (over a few nanoseconds), coupled with thermal expansion effects common to all materials, provokes inertia effects, vibrations, and propagation of stress waves. Since the linearelastic limit stress of pure lead is very low (a few MPa), two kinds of stress wave arise: elastic waves (traveling at the speed of sound in the material) and plastic waves (traveling slower). The details on these thermo-mechanical phenomena and their implications on the target response will be the object of a future publication. The robustness of the lead target when subjected to these phenomena has been tested with a beam irradiation experiment in the CERN's HiRadMat facility, described in Section 5.

The target will be equipped with mineral-insulated type-K thermocouples to monitor temperature and detect unforeseen changes in the thermal response during operation. The thermocouples will be applied on three points on the surface of the second, third, and sixth lead slice (along the beam direction), close to the spots of highest temperature (the theoretical beam axis). There will be two thermocouples for each of the three points, for a total of six sensors, to have a double measurement for each point. The tip of each thermocouple will be pushed against the lead surface by springs, and their Inconel sheath will be brazed into the stainless-steel feed-through mounted on the external stainless-steel vessel. 


\section{Beam irradiation test}

A small-scale prototype of Target \#3 has been manufactured and tested under beam irradiation in the CERN's HiRadMat facility [16]. The scope of the facility is the experimental observation of the response of materials when subjected to high-intensity particle-beam pulses. Samples of different materials or prototypes of beam intercepting devices can be temporarily installed in the facility, equipped with different measuring instruments and sensors to measure their response.

The particle beam is supplied by the Super Proton Synchrotron (SPS) ring of the CERN's accelerator complex, with a momentum of $440 \mathrm{GeV} / c$. The impacting pulse can include up to 288 bunches of $1.3 \times 10^{11}$ protons spaced by $25 \mathrm{~ns}$, totaling a pulse intensity of $3.7 \times 10^{15}$ in $7.7 \mu \mathrm{s}$. The beam energy is a factor 12 higher than the $\mathrm{n}_{-} \mathrm{TOF}$ beam and the maximum beam size is a factor 3.75 smaller. For this reason, the intensity required to test the Target \#3 prototype was quite lower than the maximum available: the prototype has been impacted with 1500 single bunches ranging between 4 and $4.5 \times 10^{10}$ protons.

The irradiated prototype consists of a series of six lead blocks and is shown in Fig. 8.

The second block along the beam direction has a size of $8 \times 8 \times 15 \mathrm{~cm}^{3}$ while the other five blocks have a size of $8 \times 8 \times 5 \mathrm{~cm}^{3}$. The blocks are supported by an Al-6082 structure and contained in a vessel of the same aluminum alloy filled with nitrogen, such that the lead blocks of the prototype are in the same environment of the new target.

As mentioned in Section 3, a prototype of the Ti-6Al-4V-cladded solution has also been tested. This second prototype consists of a lead cylinder $(\varnothing 15 \times 30 \mathrm{~cm})$ contained in a 2-mm thick Ti-6Al-4V container. The lids of the Ti-6Al-4V container are $500 \mu \mathrm{m}$ thick and are stiffened by two $15-\mathrm{mm}$ thick beryllium plates. This core assembly is contained in a stainless-steel water vessel so that it can be actively cooled by the water supply in the experimental area. The two prototypes, when installed in the irradiation area, must be contained in an aluminium tank due to radio-protection, handling, and safety rules.

The experiment on the first prototype (lead blocks in nitrogen) had the objective of studying, by post irradiation analyses, the effects in the $\mathrm{Pb}$ blocks due to repeated beam impacts (presence of voids, defects, etc.) at temperatures between 100 and $140^{\circ} \mathrm{C}$. The temperature in each block has been kept steady at the desired values thanks to a series of heating foils applied to the vessel, and monitored by thermocouples applied to each block.

The experiment on the second prototype (Ti-6Al-4V-cladded $\mathrm{Pb}$ ) had the objective of testing the ability of the Ti-6Al-4V container to resist repeated beam pulses and to measure the dynamic response of the core. The prototype has been impacted by 1000 pulses, each composed of $3.6 \times 10^{12}$ protons. A series of 27 strain gauges has been applied on the Ti-6Al-4V envelope, specifically equipped with a protective agent to resist the water flow of the active cooling system. Thanks to the strain gauges, it was possible to measure the strain distribution on the prototype so that the numerical models could be benchmarked. The details on the correlation between thermomechanical studies and measured results will be the object of future publications.

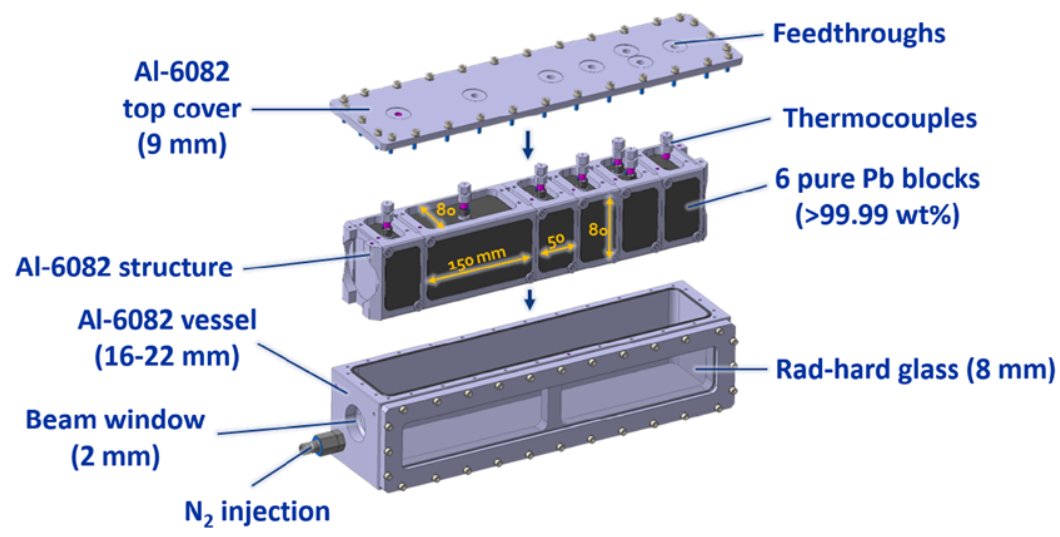

Fig. 8. Small-scale prototype of the Target \#3 final design, tested under beam irradiation in the CERN's HiRadMat facility. 
The lead blocks of the first prototype have been examined by neutron tomography at the Paul Scherrer Institut (PSI) in Switzerland to detect the onset of damage and voids hidden in the bulk of the material. The tomographic examination did not show any material damage. The lead blocks will then be subject to ultrasonic tests and destructive inspections with microstructural examinations.

Once the residual dose rate will be low enough, the second prototype (Ti-6Al-4V-cladded $\mathrm{Pb}$ ) will also be examined by neutron tomography and destructive inspections: the core will be extracted from the water vessel and the bulk of the lead cylinder will be inspected to detect damage in the material and changes in microstructure.

\section{Conclusions and future work}

After three years of design and R\&D activities, many possible solutions evaluated, and four project reviews, the third-generation neutron spallation target for the n_TOF facility at CERN is entering in the production stage in 2019. The design studies led to a target concept based on $99.9+$ wt $\%$ pure lead blocks, supported by an aluminum 6082-T6 structure and cooled by gaseous nitrogen. The assembly will be enclosed in a stainless-steel 316L vessel and equipped with water moderators with aluminum 5083 casings, one for each experimental area. The temperature of the target's lead core will be monitored by opportunely mounted thermocouples. All the critical aspects of the design, like the explosion-bonded bimetallic transition joining the stainless-steel 316L vessel to the aluminum 5083 moderator casing, have been prototyped, tested, and validated. The dynamic response of the target core, when impacted by high-intensity proton-beam pulses, has been studied and tested in a beam-irradiation experiment in the CERN's HiRadMat facility, both for the baseline design concept and for an alternative titanium-cladded solution of the target core.

The production of the target will continue during 2020. The installation of the target is planned for the same year, and commissioning with beam will take place in 2021.

Future publications on the detailed thermo-mechanical studies performed on the target are planned. The aspects of main scientific interest include:

- the high-strain rate response of the lead core subjected to impulsive thermal load and its implementation in numerical models;

- the low-strain rate plastic flow of the lead core due to creep effects and its implementation in numerical models;

- the preparation of the beam irradiation tests, the experimental results and their correlation with numerical models.

\section{References}

[1] S. Amaducci et al., Measurement of the ${ }^{235} \mathrm{U}(\mathrm{n}, \mathrm{f})$ cross section relative to the ${ }^{6} \mathrm{Li}(\mathrm{n}, \mathrm{t})$ and ${ }^{10} \mathrm{~B}(\mathrm{n}, \alpha)$ standards from thermal to $170 \mathrm{keV}$ neutron energy range at n_TOF, Eur. Phys. J. A 55(7) (2019), Art no. 120. doi:10.1140/epja/i2019-12802-7.

[2] S. Andriamonje et al., Neutron TOF Facility (PS 213) Technical Design Report, CERN, Geneva, Switzerland, CERN/INTC/2000-004, 2000.

[3] S. Andriamonje et al., Commissioning of the new spallation target for the n_TOF facility at CERN, in: Proc. Int. Topical Meeting Nucl. Res. Appl. Utilization Accel, Vienna, Austria, AT/INT-05, 2009.

[4] M. Barbagallo et al., High-accuracy determination of the neutron flux at n_TOF, Eur. Phys. J. A 49(12) (2013), Art no. 156. doi:10.1140/ epja/i2013-13156-x.

[5] M. Barbagallo et al., ${ }^{7} \mathrm{Be}(\mathrm{n}, \alpha){ }^{4} \mathrm{He}$ reaction and the cosmological lithium problem: Measurement of the cross section in a wide energy range at n_TOF at CERN, Phys. Rev. Lett. 117(15) (2016), Art no. 152701. doi:10.1103/PhysRevLett.117.152701.

[6] T.T. Böhlen et al., The FLUKA code: Developments and challenges for high energy and medical applications, Nucl. Data Sheets 120 (2014), 211-214. doi:10.1016/j.nds.2014.07.049.

[7] C. Borcea et al., Results from the commissioning of the n_TOF spallation neutron source at CERN, Nucl. Instrum. Methods Phys. Res. A 513 (2003), 524-537. doi:10.1016/S0168-9002(03)02072-2. 
[8] J. Busom Descarrega et al., Application of hot isostatic pressing (HIP) technology to diffusion bond refractory metals for proton beam targets and absorbers at CERN, Mater. Des. Process. Commun. 1(5) (2019). doi:10.1002/mdp2.101.

[9] M. Calviani et al., The n_TOF facility at CERN: Present status and future upgrades, in: Proc. 19th Int. Collaboration Adv. Neutron Sources (ICANS XIX), Grindelwald, Switzerland, 2010.

[10] M. Calviani et al., Preliminary Conceptual Design of the N_TOF Spallation Target \#3, CERN, Geneva, Switzerland, Tech. Note TOFTAR-EN-0001 (EDMS 1580996), 2016.

[11] M. Calviani et al., N_TOF Target 3 Project Status Report - Preliminary Design Review, CERN, Geneva, Switzerland, Eng. Spec. TOFTAR-ES-0002 (EDMS 1822240), 2017.

[12] M. Calviani et al., $\mathrm{n} \_$TOF neutron spallation Target \#3 $\mathrm{N}_{2}$ cooled bare Pb core - Engineering Design Report, CERN, Geneva, Switzerland, Eng. spec. TOF-TAR-ES-0003 (EDMS 2154581), 2019.

[13] R. Catherall, ISOLDE and n_TOF consolidation, in: Proc. Chamonix 2014 Workshop LHC Perform., Geneva, Switzerland, 2014.

[14] E. Chiaveri et al., The n_TOF facility: Neutron beams for challenging future measurements at CERN, EPJ Web Conf. 146 (2017), Art no. 03001. doi:10.1051/epjconf/201714603001.

[15] L. Damone et al., ${ }^{7} \mathrm{Be}(\mathrm{n}, \mathrm{p})^{7} \mathrm{Li}$ reaction and the cosmological lithium problem: Measurement of the cross section in a wide energy range at n_TOF at CERN, Phys. Rev. Lett. 121(4) (2018), Art no. 042701. doi:10.1103/PhysRevLett.121.042701.

[16] I. Efthymiopoulos et al., HiRadMat: A new irradiation facility for material testing at CERN, in: Proc. Int. Particle Accel. Conf. (IPAC), San Sebastien, Spain, TUPS058, 2011.

[17] A. Ferrari et al., FLUKA: A Multi-particle Transport Code, CERN, Geneva, Switzerland, CERN-2005-010, 2005.

[18] F. Gunsing et al., Measurements of resolved resonances of ${ }^{232} \mathrm{Th}(\mathrm{n}, \gamma)$ at the $\mathrm{n}_{-} \mathrm{TOF}$ facility at CERN, Phys. Rev. C 85(6) (2012), Art no. 064601. doi:10.1103/PhysRevC.85.064601.

[19] F. Gunsing et al., Nuclear data activities at the n_TOF facility at CERN, Eur. Phys. J. Plus 131(10) (2016), Art no. 371. doi:10.1140/epjp/ 12016-16371-4.

[20] F. Mingrone et al., The CERN n_TOF facility: A unique tool for nuclear data measurement, EPJ Web Conf. 122 (2016), Art no. 05001. doi:10.1051/epjconf/201612205001.

[21] F. Mingrone et al., Development of a neutron imaging station at the $\mathrm{n}_{-}$TOF facility of CERN and applications to beam intercepting devices, Instruments 3(2) (2019), Art no. 32. doi:10.3390/instruments3020032.

[22] C. Rubbia et al., A High Resolution Spallation Driven Facility at the CERN-PS to Measure Neutron Cross Sections in the Interval from $1 \mathrm{eV}$ to $250 \mathrm{MeV}$, CERN, Geneva, Switzerland, CERN-LHC-98-002-EET 1998. 